\section{Tratamiento de la leucemia mieloblástica aguda con translocación (8;21) en Hospital Base Valdivia: ejemplificado en un caso clínico}

\author{
JOSÉ SALINAS LAVAL ${ }^{1}$, GERARDO ALARCÓN ${ }^{1, a}$, \\ LUIS LEYTON ${ }^{2, b}$, BLAZ LESINA $^{1,3}$
}

\section{Management and follow-up of acute myeloblastic leukemia with translocation} $(8 ; 21)$. Report of one case

We describe the management and follow-up of a 20-year-old male with acute myeloblastic leukemia with translocation $(8 ; 21)[t(8 ; 21)]$. A quantitative polymerase chain reaction for $t(8 ; 21)$ in bone marrow was performed at diagnosis and after three consolidations with high doses of cytarabine. Currently, the management of this type of leukemias has been oriented towards the early detection of relapse. The concept of minimal or measurable residual disease, as the burden of leukemia cells that persist undetected, is an important tool in the therapeutic decision and follow-up of these patients.

(Rev Med Chile 2021; 149: 945-949)

Key words: Leukemia, Myeloid, Acute; Neoplasm, Residual; Polymerase Chain Reaction.

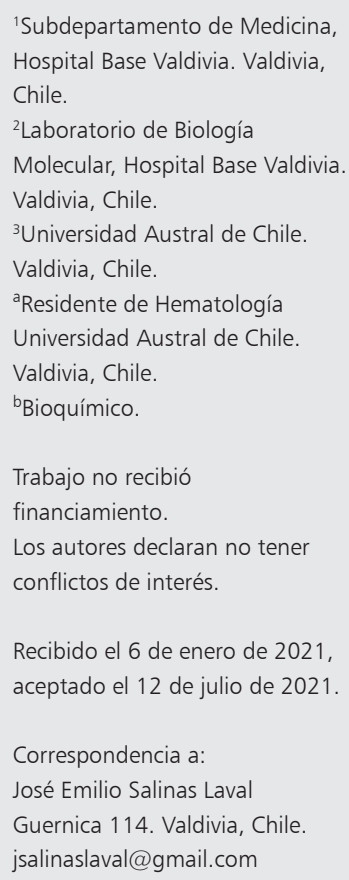

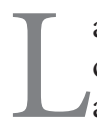
a leucemia mieloblástica aguda con translocación (8;21) (q22;q22) corresponde, junto a las leucemias mieloblásticas agudas con $\operatorname{inv}(16)(\mathrm{p} 13 \mathrm{q} 22) / \mathrm{t}(16 ; 16)$ (p13;q22), a leucemias con factor de unión nuclear o "core binding factor leukemias" $(\mathrm{CBF})^{1}$. La translocación $(8 ; 21)$ $[\mathrm{t}(8 ; 21)]$ y la inversión (16) $[\operatorname{inv}(16)]$ involucran los genes RUNX1/RUNX1T1 (AML1-ETO) y $\mathrm{CBFB} / \mathrm{MYH1} 1$, respectivamente ${ }^{2}$, dando lugar a la disrupción de un factor de transcripción heterodimérico que regula la diferenciación hematopoyética ${ }^{2}$. Estas alteraciones están presentes en 15\% de las leucemias mielobásticas agudas (LMA) ${ }^{3}$, la mitad correspondiente a $\mathrm{t}(8 ; 21)^{4}$. A pesar de estar catalogadas como LMA de buen pronóstico, la recaída ocurre en el $40 \%$ de los pacientes ${ }^{5}$ y la sobrevida a largo plazo es solo de $50 \%{ }^{6-7}$.

El estudio de otras mutaciones involucradas permite precisar de mejor manera el pronósti- co. Así, la coexpresión de la mutación del gen c-KIT (receptor de tirosin quinasa) con la t $(8 ; 21)$ confiere mal pronóstico con riesgo de recaída de $70 \%{ }^{8}$.

En Chile, la terapia estándar sigue siendo inducción con esquema a base de citarabina y una antraciclina, conocida como esquema $7+3$, que tiene una tasa de remisión completa de $87 \%$, luego se consolida con 3 ciclos de alta dosis de citarabina (HIDAC), con sobrevida libre de recaída (SLR) de $42 \%$ a 10 años ${ }^{9}$. Su sobrevida global a 5 años es de $60 \%$ en general. El uso del anticuerpo monoclonal conjugado anti CD33, gemtuzumab ozogamicin, no aumentaría la proporción de pacientes en remisión completa, pero disminuiría el riesgo de recaída y aumentaría la sobrevida global a 5 años ${ }^{10}$.

Últimamente se ha puesto énfasis en el rol de la enfermedad mínima residual (MRD) en las leucemias mieloblásticas agudas, pero su punto de 
corte, ya sea, por citometría de flujo o por biología molecular, aún es tema de debate.

Se describe el caso de un paciente con LMA $t(8 ; 21)$ tratado en el Hospital Base Valdivia. El paciente entregó su consentimiento para esta publicación.

\section{Caso clínico}

Paciente de sexo masculino de 20 años, que presentó en septiembre de 2019 faringoamigdalitis, cefalea y epistaxis abundante, de dos semanas de evolución.

Estudio diagnóstico: Hemoglobina (hb): 4,3 $\mathrm{g} / \mathrm{dl}$; reticulocitos: $0,92 \%$; leucocitosis: $35.800 \mathrm{x}$ $\mathrm{mm}^{3}$; blastos $5 \%$; recuento absoluto de neutrófilos: 15.900/ul; plaquetas: $58.000 \mathrm{x} \mathrm{mm}^{3}$. Mielograma: $72 \%$ de blastos mieloides, LMA, M2. Inmunofenotipo: $24,5 \%$ de blastos CD 13(+), CD 15(-/+), CD 33(+), CD 34(+), CD 45(-/+), CD 64(-), CD $117(+), \operatorname{MPOcy}(+)$, concluyente con leucemia mieloblástica aguda. Estudio molecular: $\mathrm{t}(8,21)$ (+), con número de copias de RUNX1/RUNX1T1 de 7790; t(15;17)(-), FlT3 ITD(-), FLT3 TKD(-), c-KIT(-). Cariograma y FISH confirman $t(8 ; 21)$.

Inició quimioterapia de inducción con citarabina $200 \mathrm{mg} / \mathrm{m}^{2}$ por 7 días en infusión continua más daunorrubicina $60 \mathrm{mg} / \mathrm{m}^{2}$ por 3 días, según protocolo nacional de drogas antineoplásicas del adulto (PANDA). Mielograma al día 29 de inducción en remisión completa, PCR cuantitativa (RTQ-PCR) t(8;21) que mostraba reducción de 2,701 logaritmos desde el diagnóstico. Se indicó 3 ciclos de consolidación con altas dosis de citarabina, $3 \mathrm{~g} /$ $\mathrm{m}^{2}$ (HiDAC) en días seguidos $(1,2,3)$ más uso de estimulante de colonias granulocíticas desde el día +8 por 5 días. El seguimiento de enfermedad mínima residual fue realizado al salir de la aplasia medular con recuento absoluto de neutrófilos (RAN) sobre $1000 /$ ul. Se tomaron muestras de aspirado medular para RT-Q-PCR $\mathrm{t}(8 ; 21)$, con una sensibilidad de $5 \times 10^{-5}$. Luego de completar las 3 consolidaciones se intercaló seguimiento molecular en médula ósea y sangre periférica cada 2 meses (Figura 1 y Tabla 1 ).

Al noveno mes de seguimiento se observó pérdida de respuesta, con aumento significativo de copias en sangre periférica, confirmado por RT-Q- PCR en médula ósea, asociado a aparición de masa retroauricular de consistencia pétrea.
Ante esta sospecha clínica de recaída extramedular, más el aumento significativo de copias RUNX1/RUNX1T1 en ambas muestras, se decidió iniciar esquema de rescate FLAG-IDA (fludarabina, idarrubicina, citarabina, filigastrim). La masa retroauricular desapareció completamente al día + 10 del protocolo FLAG-IDA. La respuesta molecular posterior a FLAG-IDA mostró reducción de transcrito a niveles menores a 150 copias en médula ósea y no detectado en sangre periférica. (Figura 1). Ante esta recaída clínica y progresión de la enfermedad mínima residual se definió trasplante de progenitores hematopoyéticos alogénico en segunda remisión completa.

\section{Discusión}

La elección de la terapia en LMA CBF tiene un impacto significativo en la tasa de remisión completa $(\mathrm{RC})$, sobrevida libre de recaída y sobrevida global (SG).

Se ha publicado que los pacientes consolidados con alta dosis de citarabina $\left(3 \mathrm{~g} / \mathrm{m}^{2}\right)$ tienen mayor beneficio en este subgrupo de $\mathrm{LMA}^{11}$. Estos datos no son del todo concluyentes en otros estudios ${ }^{12}$. Aunque en la práctica este subgrupo de pacientes se beneficia de dosis de $3 \mathrm{~g} / \mathrm{m}^{2}$, siguiendo la publicación de Byrd ${ }^{14}$; donde 3 a 4 ciclos se asociaban a sobrevida libre de leucemia (LFS) a 5 años de $71 \%$ vs $38 \%$ con un ciclo, con SG de $75 \%$ vs $44 \%$ a 5 años, el grupo alemán de LMA no demostró mejores resultados en términos de SG y SLR en relación a la dosis de citarabina y la última guía del European Leukemia Network (ELN) no hace referencia a dosis de $3 \mathrm{~g} / \mathrm{m}^{2}$ y propone citarabina $1-1,5 \mathrm{~g} / \mathrm{m}^{2}$ por 2 a 4 ciclos en riesgo citogenético favorable ${ }^{13}$.

Dada las altas dosis y sus potenciales efectos tóxicos, los autores hematólogos de esta publicación consideramos prudente dar HiDAC por 3 días sucesivos con apoyo de estimulador de colonias granulocíticas, manejo que ha comprobado ser superior en términos de recuperación hematológica (RAN> 500), reducción de infecciones y estadía hospitalaria en relación al esquema HiDAC por 3 días alternos (1-3-5) ${ }^{15-16}$.

La finalidad es dar la dosis máxima de citarabina en las consolidaciones, minimizando la toxicidad, evaluando los predictores de recaída de forma precoz. 


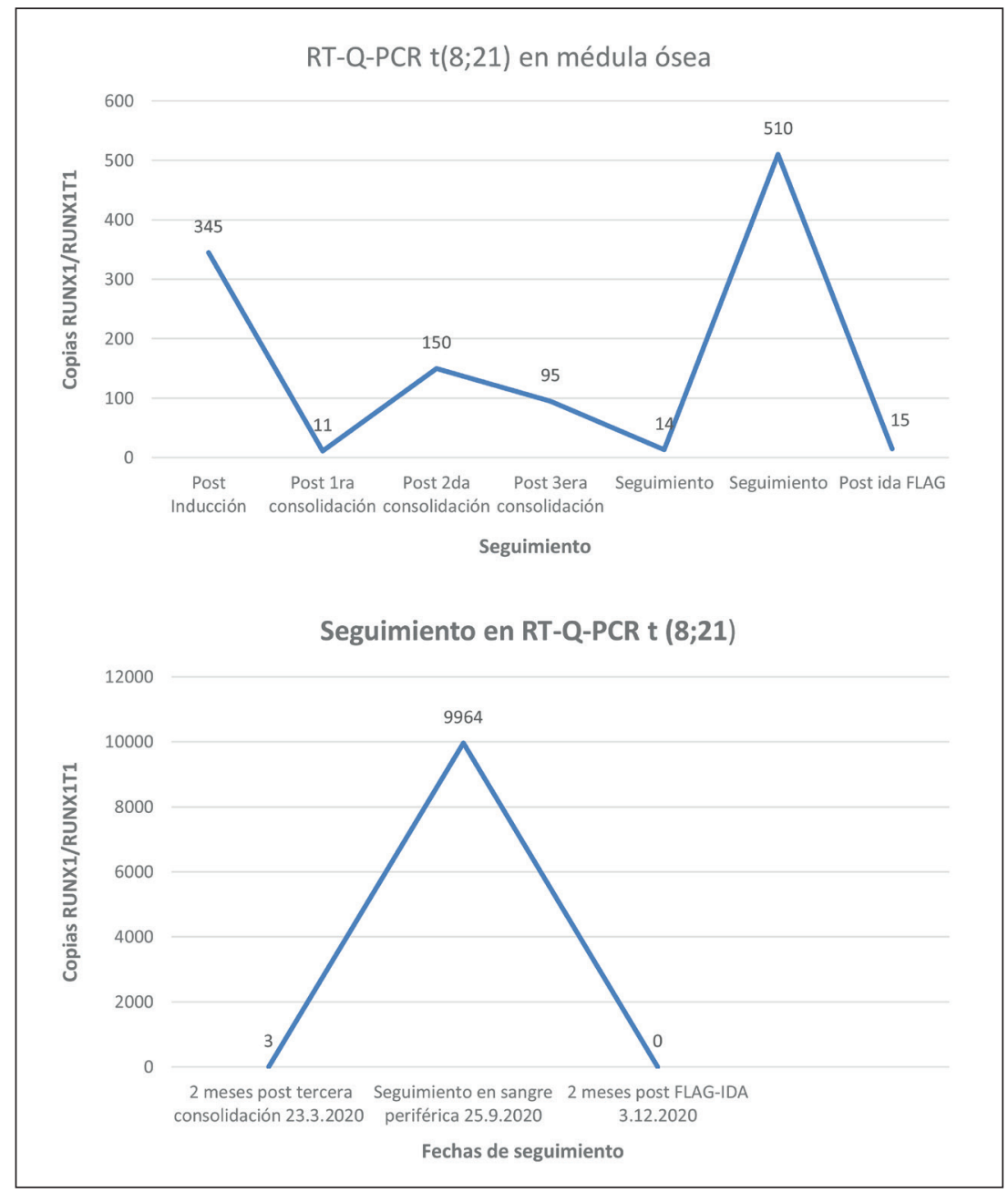

Figura 1. Copias de RUNX1/ RUNX1T1 en médula ósea y sangre periférica en diferentes puntos del seguimiento. Número de copias de RUNX1RUNX1T1 en médula ósea al diagnóstico: 7.790 .

Tabla 1. Reducción de logaritmos $(\log 10)$ en diferentes momentos del seguimiento en muestras de médula ósea

\begin{tabular}{|ccc|}
\hline $\begin{array}{c}\text { Reducción log10 desde } \\
\text { el diagnóstico en muestras } \\
\text { de médula ósea }\end{array}$ & Momento de seguimiento & Fecha \\
\hline 2,7 & Post inducción & 13.11 .2019 \\
3,9 & Post primera consolidación & 06.12 .2019 \\
3,4 & Post segunda consolidación & 13.01 .2020 \\
\hline 3,4 & Post tercera consolidación & 10.02 .2020 \\
3,4 & Seguimiento & 18.06 .2020 \\
1,5 & Seguimiento & 01.10 .2020 (confirmación de \\
\hline 3,69 & & recaída en sangre periférica) \\
\hline
\end{tabular}


Se ha publicado un score pronóstico: I-CBFIT. Este incluye edad, recuento de glóbulos blancos, mutación c-KIT y la presencia de pseudodiploidia. Los pacientes de bajo riesgo lograron $76 \%$ de SLE a 2 años, mientras que los de alto riesgo $36 \%$, proponiéndose como una herramienta útil para decidir qué pacientes deberían tratarse con esquemas más agresivo ${ }^{17}$.

Hong-Hu $\mathrm{Zhu}^{5}$ publicó un estudio prospectivo con 137 pacientes con tratamiento estratificado por riesgo según enfermedad mínima residual, definiendo respuesta molecular mayor (MMR) como el descenso de más de 3 logaritmos (>3-log) del transcrito. Los pacientes que no alcanzaron MMR posterior a la segunda consolidación, o la perdían antes de los 6 meses, eran considerados de alto riesgo e iban a trasplante de progenitores hematopoyéticos alogénico. Al comparar 69 pacientes eligibles para trasplante de progenitores hematopoyéticos alogénico con 29 pacientes que no lo recibieron, se observa una disminución de la incidencia acumulada de recaída en pacientes de alto riesgo, con $22,1 \%$ vs $78,9 \%, \mathrm{p}<0,0001$. La sobrevida libre de enfermedad en el grupo de pacientes de alto riesgo también fue mejor en el grupo sometido a trasplante alogénico $(61,7 \%$ vs $19,6 \%$ p: 0,001), así como la sobrevida global de $71,6 \%$ vs $26,7 \%$, relación que se invierte en pacientes de bajo riesgo, con una sobrevida menor en los pacientes que fueron a trasplante.

Otro estudio publicado en $2019^{18}$ concluye que lograr la reducción mayor a 2,5 logaritmos en la primera consolidación y mayor a 3 logaritmos posterior a la segunda consolidación se asocia a una disminución significativa del riesgo de recaída a 4 años. Además, lograr la MRD negativa al final del tratamiento, definido en este estudio como menos de 83 transcritos de RUNX1/RUNX1T1 en médula ósea y menos de 5 transcritos en sangre periférica, la cual predice una incidencia acumulada de recaída del $18 \%$ vs $61 \%$ en médula ósea y $23 \%$ vs $65 \%$ en sangre periférica a 4 años. Durante el seguimiento en médula ósea cada 3 meses se observó que un punto de corte para MRD > 150 transcritos de RUNX1/RUNX1T1 identifica a 77\% de los pacientes recaídos en comparación a solo $6 \%$ de recaídos bajo este punto de corte. En sangre periférica, el punto de corte que proponen es $>50$ el cual identifica a $80 \%$ de los recaídos.

Tratando de clarificar el rol de la MRD la European LeukemiaNet MRD Working Party, publicó en
$2018^{19}$ un documento de consenso en que sugieren que la MRD debe detectar células leucémicas a nivel de $0,1 \%$ ( 1 en 1.000 células). La sensibilidad del ensayo debe ser al menos de $10^{-5} \mathrm{y}$ concluyen que para pacientes con LMA $t(8 ; 21)$ no hay un punto de corte durante el tratamiento que permita hacer una recomendación de cambio de terapia.

Por lo tanto, el rol de la enfermedad mínima residual es indispensable en este subgrupo de LMA, aunque el corte, los tiempos de medición y la conducta a adoptar no están claras aún ${ }^{19}$.

Nuestro grupo plantea un seguimiento molecular cada 2 meses, una vez completado el tratamiento. Si durante la evolución se observan criterios de alto riesgo: aumento de copias $>150$ en médula ósea o $>50$ en sangre periférica, aumento de $>1$ logaritmo, conversión de MRD negativa a positiva, se debe plantear tratamiento de rescate.

Este es el primer paciente manejado en el HBV con este seguimiento, pero los autores creemos importante dar a conocer esta primera experiencia.

\section{Referencias}

1. Kuykendall A, Duployez N, Boissel N, Lancet JE, Welch JS. Acute Myeloid Leukemia: The Good, the Bad, and the Ugly. Am Soc Clin Oncol Educ Book. 2018; 38: 55573. doi: 10.1200/EDBK_199519. PMID: 30231330.

2. Speck NA, Gilliland DG. Core-binding factors in haematopoiesis and leukaemia. Nat Rev Cancer. 2002; 2 (7): 502-13. doi: 10.1038/nrc840. PMID: 12094236.

3. Creutzig U, Zimmermann M, Reinhardt D, Rasche M, von Neuhoff C, Alpermann T, et al. Changes in cytogenetics and molecular genetics in acute myeloid leukemia from childhood to adult age groups. Cancer. 2016; 122 (24): 3821-30. doi: 10.1002/cncr.30220. Epub 2016 Aug 16. PMID: 27529519.

4. Takahashi S. Current findings for recurring mutations in acute myeloid leukemia. J Hematol Oncol. 2011; 4: 36. doi: 10.1186/1756-8722-4-36. PMID: 21917154; PMCID: PMC3180439.

5. Zhu HH, Zhang XH, Qin YZ, Liu DH, Jiang H, Chen $\mathrm{H}$, et al. MRD-directed risk stratification treatment may improve outcomes of $t(8 ; 21)$ AML in the first complete remission: results from the AML05 multicenter trial. Blood. 2013; 121 (20): 4056-62. doi: 10.1182/ blood-2012-11-468348. Epub 2013 Mar 27. PMID: 23535063.

6. Grimwade D, Walker H, Oliver F, Wheatley K, Harrison C, Harrison G, Rees J, et al. The importance of diagnos- 
tic cytogenetics on outcome in AML: analysis of 1,612 patients entered into the MRC AML 10 trial. The Medical Research Council Adult and Children's Leukaemia Working Parties. Blood. 1998; 92 (7): 2322-33. PMID: 9746770.

7. Kuwatsuka Y, Miyamura K, Suzuki R, Kasai M, Maruta A, Ogawa H, et al. Hematopoietic stem cell transplantation for core binding factor acute myeloid leukemia: $\mathrm{t}(8 ; 21)$ and $\operatorname{inv}(16)$ represent different clinical outcomes. Blood. 2009; 113 (9): 2096-103. doi: 10.1182/ blood-2008-03-145862. Epub 2009 Jan 6. PMID: 19126873.

8. Paschka P, Marcucci G, Ruppert AS, Mrózek K, Chen $\mathrm{H}$, Kittles RA, et al; Cancer and Leukemia Group B. Adverse prognostic significance of KIT mutations in adult acute myeloid leukemia with inv(16) and $\mathrm{t}(8 ; 21)$ : a Cancer and Leukemia Group B Study. J Clin Oncol. 2006; 24 (24): 3904-11. doi: 10.1200/JCO.2006.06.9500. PMID: 16921041.

9. Solh M, Yohe S, Weisdorf D, Ustun C. Core-binding factor acute myeloid leukemia: Heterogeneity, monitoring, and therapy. Am J Hematol. 2014; 89 (12): 112131. doi: 10.1002/ajh.23821. Epub 2014 Aug 27. PMID: 25088818.

10. Hills RK, Castaigne S, Appelbaum FR, Delaunay J, Petersdorf S, Othus M,et al. Addition of gemtuzumab ozogamicin to induction chemotherapy in adult patients with acute myeloid leukaemia: a meta-analysis of individual patient data from randomised controlled trials. Lancet Oncol. 2014; 15 (9): 986-96. doi: 10.1016/S14702045(14)70281-5. Epub 2014 Jul 6. PMID: 25008258; PMCID: PMC4137593.

11. Bloomfield CD, Lawrence D, Byrd JC, Carroll A, Pettenati MJ, Tantravahi R, et al. Frequency of prolonged remission duration after high-dose cytarabine intensification in acute myeloid leukemia varies by cytogenetic subtype. Cancer Res. 1998; 58 (18): 4173-9. PMID: 9751631.

12. Schlenk RF, Benner A, Krauter J, Büchner T, Sauerland $\mathrm{C}$, Ehninger G, et al. Individual patient data-based meta-analysis of patients aged 16 to 60 years with core binding factor acute myeloid leukemia: a survey of the German Acute Myeloid Leukemia Intergroup. J Clin Oncol. 2004; 22 (18): 3741-50. doi: 10.1200/JCO.2004.03.012. Epub 2004 Aug 2. PMID: 15289486.

13. Döhner H, Estey E, Grimwade D, Amadori S, Appel- baum FR, Büchner T, Dombret H,et al. Diagnosis and management of AML in adults: 2017 ELN recommendations from an international expert panel. Blood. 2017; 129 (4): 424-47. doi: 10.1182/blood-2016-08-733196. Epub 2016 Nov 28. PMID: 27895058; PMCID: PMC5291965.

14. Byrd JC, Dodge RK, Carroll A, Baer MR, Edwards C, Stamberg J, et al. Patients with $\mathrm{t}(8 ; 21)(\mathrm{q} 22 ; \mathrm{q} 22)$ and acute myeloid leukemia have superior failure-free and overall survival when repetitive cycles of high-dose cytarabine are administered. J Clin Oncol. 1999; 17 (12): 3767-75. doi: 10.1200/JCO.1999.17.12.3767. PMID: 10577848 .

15. Heil G, Hoelzer D, Sanz MA, Lechner K, Liu Yin JA, Papa G, et al. A randomized, double-blind, placebo-controlled, phase III study of filgrastim in remission induction and consolidation therapy for adults with de novo acute myeloid leukemia. The International Acute Myeloid Leukemia Study Group. Blood. 1997; 90 (12): 4710-8. PMID: 9389686.

16. Jaramillo S, Benner A, Krauter J, Martin H, Kindler $\mathrm{T}$, Bentz $\mathrm{M}$, et al. Condensed versus standard schedule of high-dose cytarabine consolidation therapy with pegfilgrastim growth factor support in acute myeloid leukemia. Blood Cancer J. 2017; 7 (5): e564. doi: 10.1038/bcj.2017.45. PMID: 28548643; PMCID: PMC5518888.

17. Ustun C, Morgan E, Moodie EEM, Pullarkat S, Yeung C, Broesby-Olsen S, et al. Core-binding factor acute myeloid leukemia with $\mathrm{t}(8 ; 21)$ : Risk factors and a novel scoring system (I-CBFit). Cancer Med. 2018; 7 (9): 4447-55. doi: 10.1002/cam4.1733. Epub 2018 Aug 16. PMID: 30117318; PMCID: PMC6144246.

18. Rücker FG, Agrawal M, Corbacioglu A, Weber D, Kapp-Schwoerer S, Gaidzik VI, et al. Measurable residual disease monitoring in acute myeloid leukemia with $\mathrm{t}(8 ; 21)(\mathrm{q} 22 ; \mathrm{q} 22.1)$ : results from the AML Study Group. Blood. 2019; 134 (19): 1608-18. doi: 10.1182/ blood.2019001425. PMID: 31554635.

19. Schuurhuis GJ, Heuser M, Freeman S, Béné MC, Buccisano F, Cloos J, et al. Minimal/measurable residual disease in AML: a consensus document from the European LeukemiaNet MRD Working Party. Blood. 2018; 131 (12): 1275-91. doi: 10.1182/blood-2017-09-801498. Epub 2018 Jan 12. PMID: 29330221; PMCID: PMC5865231. 